(c) 2016 IEEE. Personal use of this material is permitted. Permission from IEEE must be obtained for all other uses, in any current or future media, including reprinting/republishing this material for advertising or promotional purposes, creating new collective works, for resale or redistribution to servers or lists, or reuse of any copyrighted component of this work in other works 


\title{
Decentralized Fault Diagnosis using Analytical Redundancy Relations: Application to a Water Distribution Network
}

\author{
Vikas Gupta and Vicenç Puig
}

\begin{abstract}
In this paper, a decentralized fault diagnosis algorithm for large scale systems is proposed. The fault diagnosis algorithm starts with obtaining a set of ARRs (analytical redundancy relations) from the system model and available sensors. These ARRs are converted into a graph that is divided into various subgraphs using a partition algorithm. From various subgraphs, different fault signature matrices are obtained. This allows designing a decentralized fault diagnosis system by using a local diagnoser for each subsystem and a global one for coordination. Entire proposed decentralized fault diagnosis algorithm is divided into five different blocks. In order to illustratethe application of the proposed algorithm, a casestudy based on the Barcelona water network is used.
\end{abstract}

\section{INTRODUCTION}

Generally a large scale system consists of lot of components, being complex and difficult to maintain a single diagnoserfor the whole system. Thus, a decentralized/distributed fault diagnosis system has been considered in place of centralized fault diagnosis system since centralized fault diagnosis system has lot of disadvantages, as e.g.ina centralized system all the information has to be collected in one location which is generally not possible. Moreover, a centralized system need a high performance centralized unit which is in most cases is not available. Due to these difficulties in recent years decentralized/distributed fault diagnosis techniques has been adopted. Decentralized diagnosis consists of both a global diagnoser and a local diagnoser working parallel to monitor and detect a fault or faults in large scale system, some of examples of decentralized diagnosis are shown in [1,2]. The large scale system is first divided into various subsystems, each subsystem has its own local diagnoser and there will be a global diagnoser which contains information about the shared variables between each subsystem. Information of such type of decentralized systemis mentioned in the literature $[3,4,5]$. Already in the past literature distributed fault diagnosis algorithms for a large scale system are present.

This work has been funded by the Spanish Government (MINECO) through the project CICYTECOCIS (ref. DPI201348243-C2-1-R), by MINECO and FEDER through the project CICYTHARCRICS (ref.DPI2014-58104-R).

Vikas Gupta and Vicenç Puig is with Advanced Control Systems (SAC) research group at Universitat Politècnica de Catalunya (UPC),Barcelona, Spain (e-mail: vikas.gupta@upc.edu).
In [12], a fault diagnosis algorithm is discussed which starts with obtaining a set of ARRs (analytical redundancy relations) from a model. After obtaining the ARRs, the partitioning of a system into subsystem is done by using fault signature matrix approach, when compared to other past literatures [6] [11] [15] most of the fault diagnosis algorithm had adopted graph approach for partition. From the past literature, it is very clear that graph approach for partition has more advantages than fault signature matrix partition approach.

In this paper, a new approach for decentralized fault diagnosis of large scale systems is presented which starts by obtaining a set of ARRs from the system model and available sensors. Then, after obtaining ARRs, the system is divided into subsystem by a graph method. After which for each subsystem a local fault signature matrix is obtained and a single global fault signature matrix is obtained for all the subsystems which contains information of shared variables between various subsystems. This allows designing a decentralized fault diagnosis system with including a local diagnoser for each subsystem and a global one for coordination.In order to demonstrate the application of the proposed algorithm, a case study based on the Barcelona drinking waternetwork (DWN) is used.

The structure of the paper is the following: Section 2 describes the proposed approach. Section 3 describes the implementation. Section 4 describes the application with example. Finally, in Section 5, conclusions are presented.

\section{PROPOSED APPROACH}

The goal of proposed approach is to obtain a set of local diagnosers that are coordinated by a global diagnoser allowing decentralised diagnosis. This approach has an off-line phase that starts by obtaining a setof analytical redundancy relations (ARRs) that can be represented in a form of a matrix $P$ from a system structural matrix $M(z, x)$ or in short form just $M$ by using ranking algorithm [15]. $M$ consists of set of constraints (equations) $z$ and variables $x$, some of them known and other unknown. Technically, to obtain the ARRs, the unknown variables are replaced by known variables of the systems. The rows of matrix $P$ correspond to the 
ARRs and columns are the measured variables. The matrix $P$ is converted into vertex and edge graph: any 1or -1 present in rows of matrix $P$ makes that particular row a vertex of the graph and all the 1 or -1 present at same location of two rows is connected edge between the vertexes. From this vertex and edge graph, small vertex and edge graph or subsystem is generated by using a partition algorithm. The first step to implement partition algorithm is to find the strongly connected vertices. A strongly connected vertex is the one which has maximum number of edges. This vertex will be the basis for forming the first subsystem being its core. Second subsystem is formed by second strongly connected vertex. The important condition is that no two subgraphs can have same vertex but same edge can be shared. Together all the subgraphs must contain all the vertices of a system, that is, no vertex must be left. Every vertex must be part of any one subsystem and the subsystem should be least connected.After this,the fault signature matrix is generated for each subsystem. Every subsystem has one local fault signature which contains unshared and shared variables and also all the subsystems have one common global fault signature matrix which contains shared variables between various subsystems. A fault signature matrix is created by converting all elements of each subsystem matrix $P_{i}$ into 0 and 1.0 is maintained as zero while all non zero elements are converted into 1 . This is the end of offline scenario in which we designed the various sub systems. Now in the next step, we consider the on-line operation in which the ARRs associated to each subsystem are employed to detect and isolatesensor faults from fault signature matrices associated to the ARRs. This is done by comparing fault signature matrix of eachsubsystem with observed fault signature matrix, column wise or variable wise. If a fault is detected it is then checked whetherthe fault is in unshared variable or shared variable. In case that the fault is affecting an unshared variable, the local diagnosers can directly isolate the fault. Otherwise, the global diagnoser has to fuse the information of the local diagnosers sharing the variable.

\section{IMPLEMENTATION}

The proposed approach can be implemented by means of five blocks (Figure 1). In this section, the description for each block is provided.

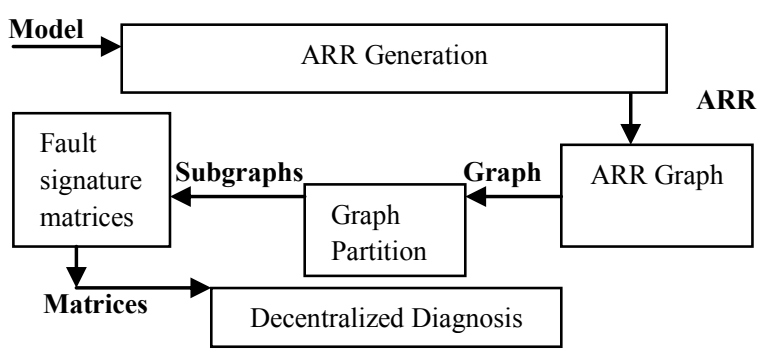

Fig 1: Various blocks of proposed algorithm

Block 1: ARR Generation

Input to the Block: The system structural matrix $M$. Output of the Block: ARRs in form of matrix $P$.

In Block 1, ARRs are obtained in form of parity matrix $P$ from asystem structural matrix $M(z, x)$ by using ranking algorithm [17]. Let $Z=\left\{z_{1}, z_{2}, z_{3}\right.$, $\left.z_{4} \ldots \ldots z_{m}\right\}$ be the set of the constraints which represent the system model and let $X=\left\{x_{1}, x_{2}, x_{3}, x_{4} \ldots \ldots x_{n}\right\}$ be the set of the variables which contains three subsets: let $K=Y \cup U$ be the set of known variables: $U$ is the subset of input variables, $Y$ is the subset of the output variables and $K$ is the subset of the unknown (nonmeasured) variables. The structure of the model is described by the binary relation:

$$
M: z \times x \rightarrow\{0,1\}
$$

where: $\left(z_{i}, x_{j}\right) \rightarrow M\left(z_{i}, x_{j}\right)=1$ if $z_{i}$ applies to $x_{j}$ and $M\left(z_{i}\right.$, $\left.x_{j}\right)=0$,otherwise.

The unknown variables are replaced by known variablesof the system model to obtain ARRs in form of parity matrix $P$.

\section{Block 2: ARR Graph Generation:}

Input to the Block: ARRs in form of parity matrix $P$

Output of the Block: ARR Graph.

Block 2 obtains the ARR graph from the set of ARRs obtained in form ofparity matrix $P$ in Block 1 . A graph is generally defined as an abstract representation of a group of objects from a collection, where few pairs of objects are joint by links. The elements which are interconnected are typicallycalled vertices while the connected links are called edges.If anylor -1 present in rows of parity matrix $P$ makes that particular row a vertex of the graph and all the 1 or -1 present at same location of two rows is connected edge between the vertexes. The ARR matrix $P$ is feed as input to Block 2. From ARR matrix $P$, the vertex and edges of graph are obtained and finally graph $G(V, E)$, where $V$ denotes the set of vertices, $E$ is the set of edgesis created which is the output of Block 2. The graph $G(V, E)$ can be represented in form of incidence matrix denoted as $I_{M}$, which is defined suchthat 
$I_{M i j}=\left\{\begin{array}{cccc}-1 & \text { if the edge } & e_{j} \text { leaves vertex } & v_{i} \\ 1 & \text { if the edge } & e_{j} \text { enters vertex } & v_{i} \\ & 0 & \text { otherwise }\end{array}\right.$

This matrix has dimensions $n_{v} \times n_{e}$, where $n_{v}$ corresponds with the total number ofvertices and $n_{e}$ denotes the total number of edges.

Block 3: Partition of ARR Graph

Input to the Block: ARR graph

Output of the Block: Partitioned ARR graph

The first step to implement partition algorithm is to find the strongly connected vertices. A stronglyconnected vertex is the one which has maximum number of edges. This vertex will be the basis for forming the first subsystem being the core of the first subsystem. Second subsystem is formed by second strongly connected vertex. The important condition is that no two subgraphs can have same vertex but same edge can be shared. Together all the subgraphs must contain all the vertices of a system, that is, no vertex must be left. Every vertex must be part of any one subsystem and the subsystem should be least connected.The maximum weight $\omega$ for each vertex is equal to number of edges each vertex have. The heaviest vertex is the vertex which has maximum number of edges, the heaviest vertex forms the first subgraph and the centre of the first subgraph $G_{l}$ is defined. Those vertices which are connected to this heaviest vertex are included in $G_{1}$ or in the first subgraph. The set of non-selected [11] vertices are defined as $V_{r}=\left\{v_{j} \in V: v_{j} \notin V_{l}\right\}$. The above procedure is repeated for all vertices $v_{\mathrm{j}} \in V_{r}\left(\mathrm{j}=\left\{1,2, \ldots, n_{v}\right\}\right)$ until $V_{r}$ is empty. The subgraph of higher connectivity is highlighted by the above method.The subgraphs which have only one vertex are merged to theclosest subgraph and thus a set of subgraphs $G_{i}\left(V_{i}, E_{i}\right)$, for $i=1,2$. . $k$, is obtained

Algorithm for Block 3:

1: $I M \leftarrow$ System topology

2: $G(V, E) \leftarrow I M$

3: for $j=1$ to $n_{v}$ do

4: Compute $\omega_{j}$ as the number of edges each vertex have

5: end for

6: $V_{r} \leftarrow V, i=1$

7: repeat

8: Find $v \in V_{r}$ with maximum $\omega$

9: $V_{i} \leftarrow v$ and all its neighbour vertices

10: $V_{r}=V-\left\{\bigcup_{h=1}^{i} V_{h}\right\}$
$11: i=i+1$

12: until $V_{r}=\varnothing$

Block 4: Fault Signature Matrices Formation Input to the Block: Partitioned ARR graph

Output of the Block: A set of fault signatures matrices, one for each subgraph

ARRs obtained in Block 1 are constraints that only involve known parameter $\theta$ andmeasured [7] variables $(y, u)$. The set of ARRs are represented as

$$
R=\left\{r_{i} \mid r_{i}=\Psi_{i}\left(y_{k}, u_{k}, \theta_{k}\right), i=1, \ldots, n_{\tau}\right\}
$$

$\psi_{i}$ is the mathematical expression for ARRs and $n_{r}$ is theARRs number obtained. Fault diagnosis is done by identifying the set of consistent ARRs

$$
R_{0}=\left\{r_{i} \mid r_{i}=\Psi_{i}\left(y_{k}, u_{k}, \theta_{k}\right),=0, i=1, \ldots, n_{\tau}\right\}
$$

and inconsistent ARRs

$$
R_{1}=\left\{r_{i} \mid r_{i}=\Psi_{i}\left(y_{k}, u_{k}, \theta_{k}\right), \neq 0, i=1, \ldots, n_{\tau}\right\}
$$

when some inconsistency in (2)at time instant $k$ is detected, the process of fault isolation starts byobtaining the observed fault signature, where each single fault signal indicator $\phi_{i}(k)$ is defined as follows:

$$
\phi_{i}(k)=\left\{\begin{array}{lll}
0 & \text { if } & r_{i}(k) \in R_{0}, \\
1 & \text { if } & r_{i}(k) \in R_{1} .
\end{array}\right.
$$

Fault isolation is the binary relation between the considered fault hypothesis set $\left\{f_{1}(k), f_{2}(k) \ldots \ldots . f_{n f}\right.$ $(k)\}$ and the fault signal indicators $\phi_{i}(k)$, stored in the Fault Signature Matrix $F$. The fault hypothesis $f_{j}$ is expected to affect the residual $r_{i}$ when $F_{i j}$, is equal to 1 and in such case the related fault signal $\phi_{i}(k)$ is equal to 1 , means this fault is affecting the monitored system, otherwise, the element $F_{i j}$ is zero-valued. A column of this matrix is known as a theoretical fault signature. The fault isolation starts by finding a match between the observed fault signatures with some of theoretical fault signatures.

\section{Block 5: Decentralized Fault Diagnosis}

Input to the Block: Various fault signature matrices corresponding to the different subsystems

Output of the Block: Diagnosis faults present in the subsystems

Till Block 4 the algorithm is operated in an offline scenario where various local fault signature matrices and a global fault signature matrix are obtained from the designed of the system. Block 5 is completely operated in an online scenario where several diagnosers obtained in Block 4 are working parallel in a large scale system and continuously comparing observed fault signature matrices of each subsystem with its original fault signature matrix. In this part of 
the algorithm, fault detection and isolation is done using concept of agent using traditional FDI approach. This part of the algorithm is online while rest of the algorithm work in offline conditions. Each subsystem is represented by an agent $A_{1}, A_{2}, \ldots, A_{n}$ and the global coordinator or diagnoser which contains information of all shared variables of each subsystem is represented by an agent $G$. The agents of each subsystem communicate with agent of global coordinator or diagnoser in form of messages to detect and isolate a given fault or faults in their respective subsystem. Actually the entire process three separate parts, In the first part, each agent does local diagnosis to detect any faulty ARR in its system and if any ARR is faulty, whether fault occur in a shared variable or unshared variable or in both, if the fault occur in shared variable or unshared variable connected with shared variable than the agent of a subsystem sends a message containing faulty shared variable or variables number to agent of global coordinator. In the second part, when the global coordinator or diagnoser receives the information about the faulty candidates, the agent diagnoses to find that whether the shared variable or variables are faulty or not. If the agent finds that the faulty candidates are also faulty candidate or candidates in his subsystem, it pass the information to the given agent from whom he receives the information in form of data or number of that shared variable or variables otherwise it sends 00 data to the agent from whom he

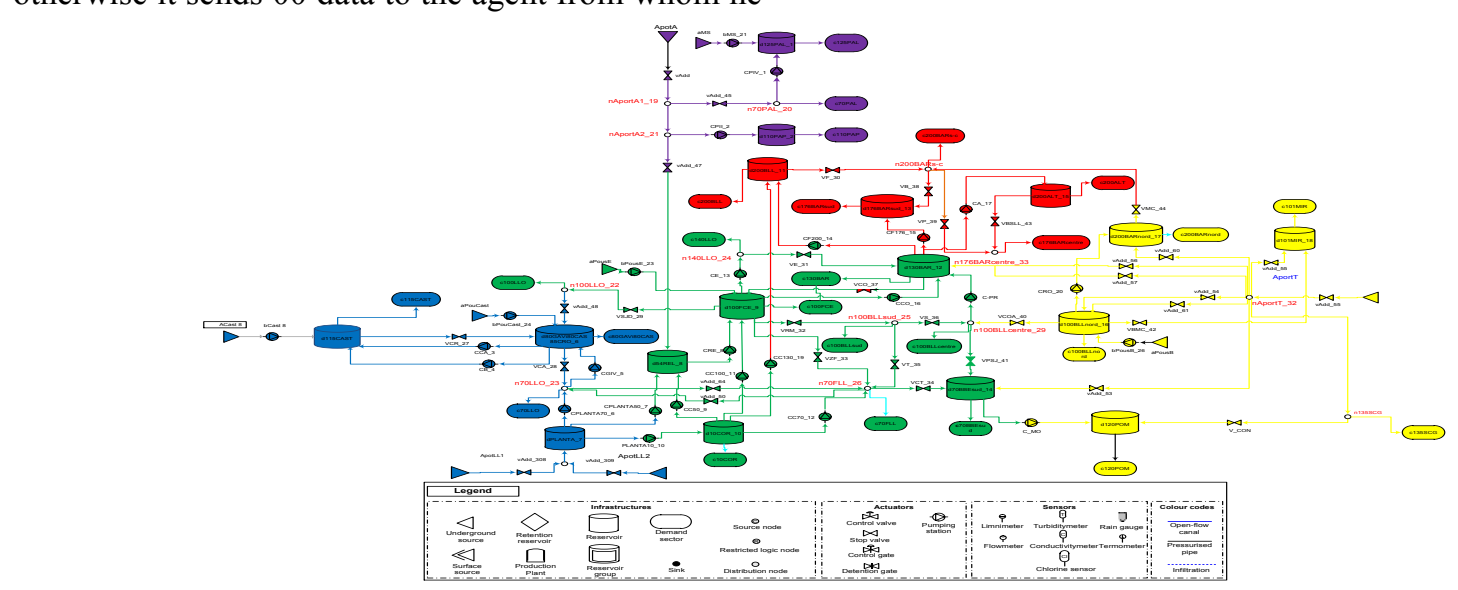

Figure 2: Barcelona water network receives the message indicating that this particular candidate or candidates do not belong to his subsystem or in short are not faulty. In the third part, which is the main part, controls sending of messages from each agent and receiving information by agent of global coordinator, on the basis of received information final computation is done to detect which variable or variables are faulty.

\section{APPLiCATION EXAMPLE}

The proposed algorithm is implemented on Barcelona water network shown in Figure 2. The proposed fault diagnosis algorithm starts from discrete-time space state model (for more details see [9][10]):

$x(k+1)=A x(k)+B_{u} u(k)+B_{p} d(k)(5)$

$y(k)=C x(k)$

where $A \in R^{n x n}, B_{u} \in R^{n x m}, C \in R^{r x n}$ are the state space matrices and $B_{p} \in$ is $R^{n x p}$ the disturbance known, $x \in$ $R^{n}$ is the state vector corresponding to the volume of deposits, $u \in R^{m}$ is the vector of input variables, $d \in$ $R^{p}$ corresponds the vector of known disturbances, in this case are the water demands, $y \in R^{r}$ is the vector of outputs.

\section{ARR Generation and Graph (Block 1 and 2)}

ARR generation algorithm in Block 1 applied to (5) produces set of ARRs in form of matrix $P$. Starting with this matrix, Block 2 produces the ARR graph presented in Figure 3. 


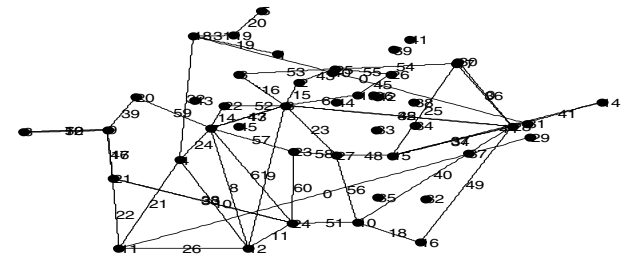

Figure 3. ARR graph

ARR graph partition (Block 3)

Block 3 partitions the graph in subgraphs (subsystems) that are presented in different colours in Figure 4 and in the orginal water network scheme in Figure 2. Table I summarizes the number of ARRs and shared variables of each subsystm.

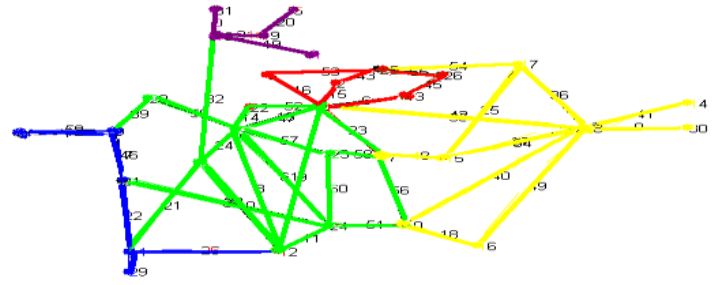

Figure 4: Subgraphs of Barcelona city water network

\begin{tabular}{llcc} 
Number & Color & \# ARRs & \# Shared variables \\
\hline 1 & purple & 4 & 1 \\
\hline 2 & red & 5 & 4 \\
\hline 3 & yellow & 7 & 6 \\
\hline 4 & green7 & 1 & 3 \\
\hline 5 & blue & 5 & 4 \\
& Table I. Barcelona DWN subsystems
\end{tabular}

\section{Fault signature matrices (Block 4)}

Figure 7.1 to 7.5 presents the local fault signatures matrices associated to each subystem. In this tables, unshared variables are presented in green and blue while shared variables in yellow. Faults associated to this variables can be diagnosed at local level only in case of green variables. Faults in blue and yellow variables can only be diagnosed at global level since they correspond to a share variable (yellow) or correspond to a variable (blue) that participates in an ARR including shared variables. Figure 8 presents the table of shared varibles between subsystems used by the global diagnoser.

\section{Decentralized fault diagnosis (Block 5)}

Finally, to illustrate the on-line Block 5, suppose that a fault occurs in ARR 5 of $1^{\text {st }}$ subsystem (indicated in red color) in Figure 7.1, in particular in variable 20. Since 20 is unshared variable, the fault can be detected and isolated at local level and only local fault signature matrix has to be consulted but suppose fault occures in ARR 18 of $1^{\text {st }}$ subsystem (indicated in red color) in Figure 7.1 and the fault occurs in variables 31 and 32 . The variable 32 is global or shared and variable 31 is though local or unshared variable but connected to 32 which is a global or shared varible betwwen $1^{\text {st }}$ and $4^{\text {th }}$ subsystem, so to detect and isolate fault in 31 and as well as 32, both local and global fault signature matrices has to be consulted.

\section{CONCLUSIONS}

In this paper a decentralized fault diagnosis technique is presented. First from a system structural model, ARRs are obtained. From ARRs, a vertex and edge graph is generated; this vertex and edge graph is subdivided into various subgraphs or subsystem through partition algorithm. For each subgraph a local fault signature matrix is generated which contain both local and shared variables of subgraph and also a global fault signature matrix is generated which contain information of only shared variables of all subgraphs. Then using observer method the fault signature matrix of original subsystem is compared with observed matrix of that subsystem, if a fault or faults are present, than it is checked whether fault is in unshared variable or shared variable of the subsystem.

\section{REFERENCES}

[1] L. Console, C. Picardi, and D. TheseiderDuprè. A framework for decentralized qualitative model-based diagnosis. In International Joint Conference on Artificial Intelligence (IJCAI), pages 286-291, Hyderabad, India, 2007.

[2] Y. Pencolé and M.-O. Cordier. A formal framework for the decentralised diagnosis of large scale discrete event systems and its application to telecommunication networks. Artificial Intelligence, 164(1-2):121-170, 2005.

[3] F. Boem, R.M.G. Ferrari, T. Parisini, and M. M. Polycarpou. Distributed fault diagnosis for continuous time nonlinear systems: The input-output case. Annual Reviews in Control, 37(1):163 - 169, 2013.

[4] J. Biteus, E. Frisk, and M. Nyberg. Distributed diagnosis using a condensed representation of diagnoses with application to an automotive vehicle. IEEE Transactions on Systems, Man, and Cybernetics - Part A: Systems and Humans, 41(6):1262-1267, November 2011.

[5] I. Roychoudhury, G. Biswas, and X. Koutsoukos. Designing distributed diagnosers for complex continuous systems. IEEE Transactions on Automation Science and Engineering, 6(2):277-290, April 2009

[6] S. Indra L. Travé-Massuyès, E. Chanthery. Decentralized Diagnosis with Isolation on Request for Spacecraft.8th IFAC Symposium on Fault Detection, Supervision and Safety of Technical Processes, vol 8, part 1, pages 283-288, 2012.

[7] V. Puig, C. Ocampo-Martinez. Decentralised fault diagnosis of large-scale systems: Application to water transport networks. $26^{\text {th }}$ International Workshop on Principles of Diagnosis, 2015. 
[8] S. Indra, L. Travé-Massuyès. Spacecraft fault Detection and Isolation system design using decentralized analytical redundancy. Advances in Aerospace Guidance, Navigation and Control. Springer, pages 247-263, 2013.

[9] C. Ocampo-Martinez, V. Puig, G. Cembrano, R. Creus,and M. Minoves. Improving water management efficiency by using optimization-based control strategies: the Barcelona case study. Water Science \& Technology: Water supply, 9(5):565-575, 2009.

[10] C. Ocampo-Martinez, V. Puig, G. Cembrano, and J. Quevedo. Application of predictive control strategies to the management of complex networks in the urban water cycle [applications of control]. IEEE Control Systems Magazine, 33(1):15-41, 2013.

[11] C. Ocampo-Martinez, S. Bovo, and V. Puig. Partitioning approach oriented to the decentralised predictive control of large-scale systems. Journal of Process Control, 21(5):775786, 2011.

[12] A. L.Gehin, B.Ould Bouamama. Design of distributed Fault Detection and Isolation systems.9th IFAC Symposium on Fault Detection, Supervision and Safety of Technical Processes, September 2-4, 2015.

[13] S. Tornil-Sin, C. Ocampo-Martinez, V. Puig, and T. Escobet. Robust fault diagnosis of nonlinear systems using interval constraint satisfaction and analytical redundancy relations. IEEE Transactions on Systems, Man, and Cybernetics: Systems, 44(1):18-29, Jan 2014.

[14] R. Ferrari, Distributed Fault Detection and Isolation of Large-scale Nonlinear Systems: an Adaptive Approximation Approach, pages 1- 133, 2007.

[15] M.Blanke,M. Kinnaert, J. Lunze, M. Staroswiecki. Diagnosis and Fault-Tolerant Control. Springer. 3th Edition, 2016.

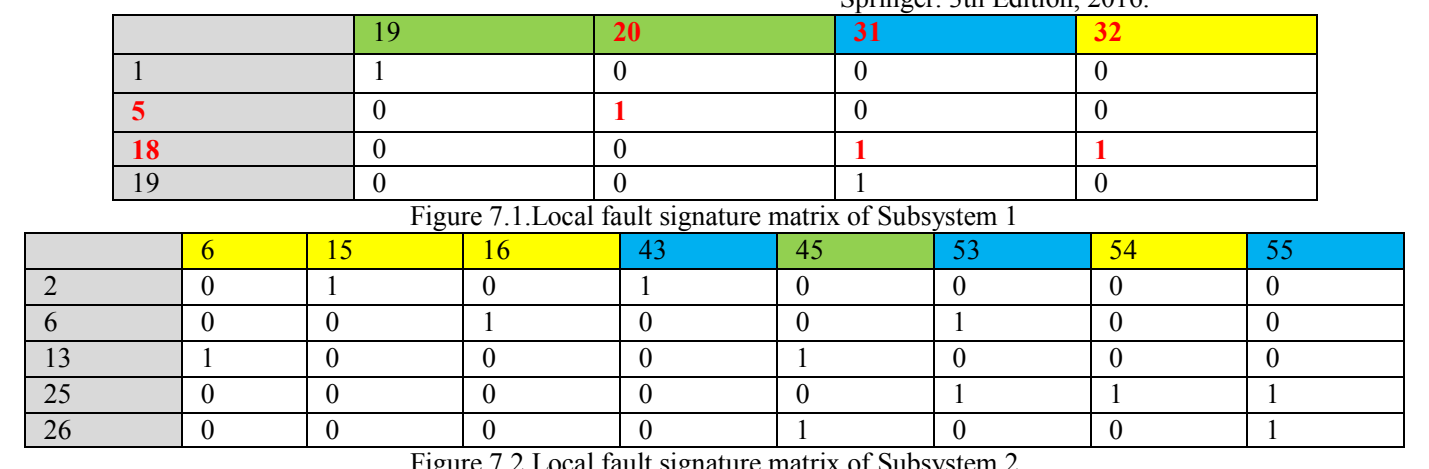

\begin{tabular}{|l|l|l|l|l|l|l|l|l|l|l|l|l|l|l|l|l|l|l|l|l|l|l|l|l|l|}
\hline & 6 & 8 & 9 & 10 & 11 & 13 & 14 & 15 & 16 & 23 & 24 & 26 & $\mathbf{3 2}$ & 33 & 35 & 38 & 42 & 47 & 51 & 52 & 57 & 58 & 59 & 60 & 61 \\
\hline 3 & 1 & 0 & 1 & 0 & 0 & 1 & 0 & 1 & 1 & 1 & 0 & 0 & 0 & 0 & 1 & 0 & 1 & 1 & 0 & 1 & 0 & 0 & 0 & 0 & 0 \\
\hline 4 & 0 & 0 & 0 & 1 & 0 & 0 & 0 & 0 & 0 & 0 & 1 & 0 & 1 & 0 & 0 & 0 & 0 & 0 & 0 & 0 & 0 & 0 & 0 & 0 & 0 \\
\hline 7 & 0 & 1 & 0 & 0 & 0 & 1 & 0 & 0 & 0 & 0 & 1 & 0 & 0 & 0 & 0 & 0 & 0 & 1 & 0 & 0 & 1 & 0 & 1 & 0 & 1 \\
\hline 12 & 0 & 1 & 1 & 1 & 1 & 0 & 0 & 0 & 0 & 0 & 0 & 1 & 0 & 0 & 0 & 0 & 0 & 0 & 0 & 0 & 0 & 0 & 0 & 0 & 0 \\
\hline 22 & 0 & 0 & 0 & 0 & 0 & 0 & 1 & 0 & 0 & 0 & 0 & 0 & 0 & 0 & 0 & 0 & 0 & 0 & 0 & 0 & 0 & 0 & 0 & 0 & 0 \\
\hline 23 & 0 & 0 & 0 & 0 & 0 & 0 & 0 & 0 & 0 & 0 & 0 & 0 & 0 & 0 & 0 & 0 & 0 & 0 & 0 & 0 & 1 & 1 & 0 & 1 & 0 \\
\hline 24 & 0 & 0 & 0 & 0 & 1 & 0 & 0 & 0 & 0 & 0 & 0 & 0 & 1 & 1 & 0 & 1 & 0 & 0 & 1 & 0 & 0 & 0 & 0 & 1 & 1 \\
\hline
\end{tabular}

Figure 7.4 Local fault signature matrix of Subsystem 4

\begin{tabular}{|l|l|l|l|l|l|l|l|l|l|l|l|l|l|l|l|}
\hline \multicolumn{10}{|c|}{ Figure 7.4 Local fault signature matrix of Subsystem 4} & 46 & 50 & 59 \\
\hline 8 & 7 & 12 & 17 & 21 & 22 & 26 & 33 & 38 & 39 & 46 & 0 \\
\hline 9 & 1 & 1 & 1 & 0 & 0 & 0 & 0 & 0 & 0 & 0 & 0 & 1 & 1 & 1 & 0 \\
\hline 11 & 0 & 0 & 0 & 1 & 1 & 1 & 0 & 0 & 0 & 0 & 0 & 0 \\
\hline 20 & 0 & 0 & 0 & 0 & 0 & 0 & 0 & 0 & 1 & 0 & 0 & 1 \\
\hline 21 & 0 & 0 & 1 & 0 & 1 & 0 & 1 & 1 & 0 & 1 & 0 & 0 \\
\hline
\end{tabular}

Figure 7.5 Local fault signature matrix of Subsystem 5

\begin{tabular}{|c|c|c|c|c|c|c|c|c|c|c|c|c|c|c|}
\hline & 6 & 15 & 16 & 23 & 26 & 32 & 33 & 35 & 38 & 42 & 51 & 54 & 58 & 59 \\
\hline $\mathbf{1}$ & 0 & 0 & 0 & 0 & 0 & 1 & 0 & 0 & 0 & 0 & 0 & 0 & 0 & 0 \\
\hline $\mathbf{2}$ & 1 & 1 & 1 & 0 & 0 & 0 & 0 & 0 & 0 & 0 & 0 & 1 & 0 & 0 \\
\hline $\mathbf{3}$ & 0 & 0 & 0 & 1 & 0 & 0 & 0 & 1 & 0 & 1 & 1 & 1 & 1 & 0 \\
\hline $\mathbf{4}$ & 1 & 1 & 1 & 1 & 1 & 1 & 1 & 1 & 1 & 1 & 1 & 0 & 1 & 1 \\
\hline $\mathbf{5}$ & 0 & 0 & 0 & 0 & 1 & 0 & 1 & 0 & 1 & 0 & 0 & 0 & 0 & 1 \\
\hline
\end{tabular}

Figure 8: Table shared variablesof Barcelona water network 